Issaka OUÉDRAOGO ${ }^{1}$

Blandine Marie Ivette NACOULMA ${ }^{1}$

Oumarou OUÉDRAOGO ${ }^{1}$

\section{Karen $\mathrm{HAHN}^{2}$}

Adjima ThIOMBıANo ${ }^{1}$

${ }^{1}$ Université de Ouagadougou

Ufr/Svt

Laboratoire de biologie et écologie végétales

03 BP 7021 Ouagadougou 03

Burkina Faso

2 J. W. Goethe University

Institute for Ecology, Evolution and Diversity

Max-von-Laue-Str. 13

Hauspostfach 19

60438 Francfort-sur-le-Main

Allemagne

\title{
Productivité et valeur économique des calices de Bombax costatum Pellegr. \& Vuillet en zone soudanienne du Burkina Faso
}

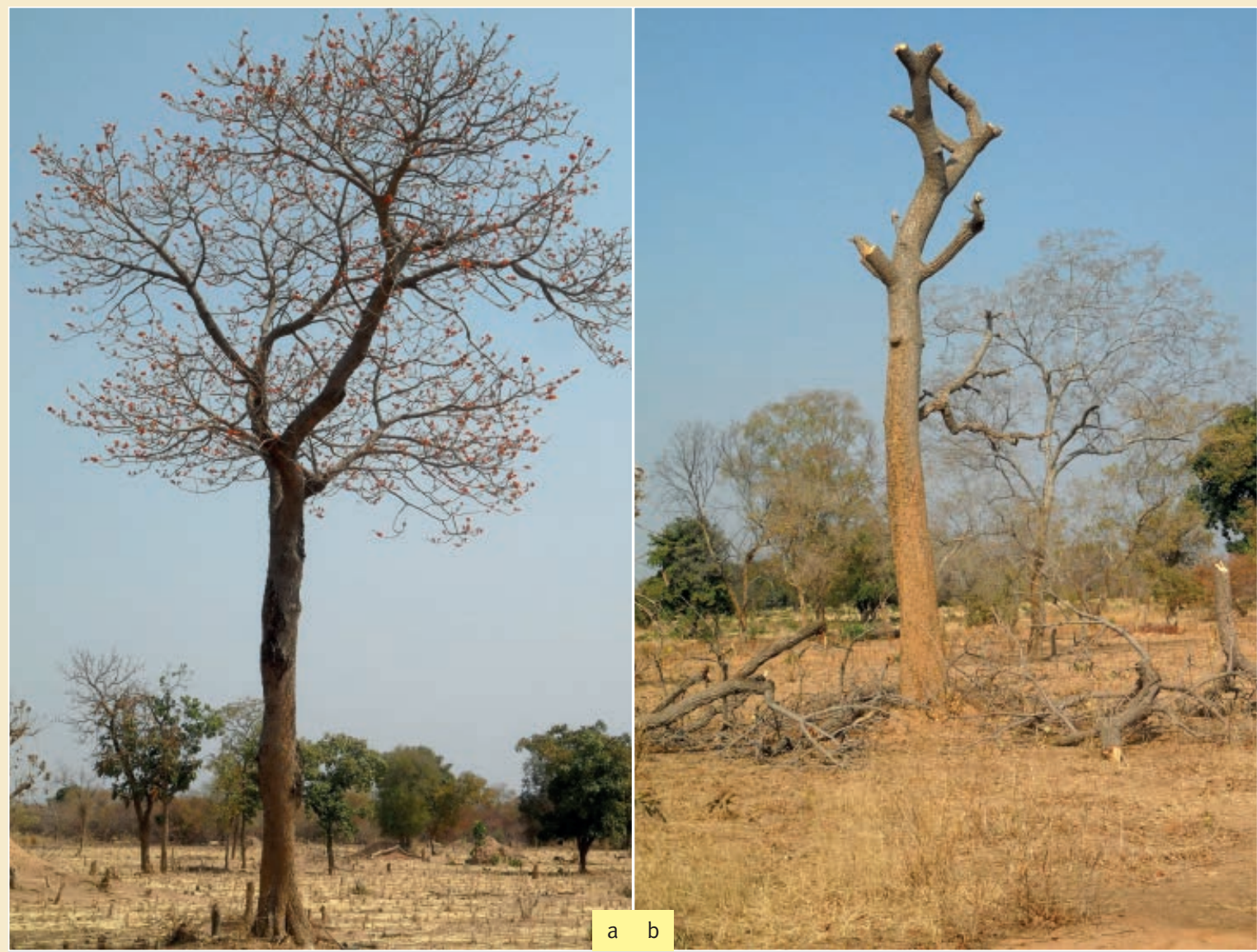

Photos 1.

a : pied de $B$. costatum avant récolte des fleurs ; b : pied après récolte des fleurs. Photos I. Ouédraogo. 


\section{RÉSUMÉ}

\section{PRODUCTIVITÉ ET VALEUR ÉCONOMIQUE DES CALICES DE BOMBAX COSTATUM PELLEGR. \& VUILLET EN ZONE SOUDANIENNE DU BURKINA FASO}

Arbre des savanes et des forêts claires sahélo-soudaniennes à guinéennes, Bombax costatum est une espèce fortement exploitée pour ses calices et menacée dans plusieurs localités au Burkina Faso. Une alternative à la conservation résiderait dans la reconnaissance de l'intérêt économique de l'espèce. Cette étude évalue la valeur monétaire de la productivité en calices de $B$. costatum pour fournir des arguments nécessaires à sa conservation. Pour ce faire, des individus de B. costatum ont été échantillonnés dans trois modes d'utilisation des terres; leur production florale a été intégralement récoltée afin d'évaluer la quantité de calices produite. Des modèles allométriques ont été élaborés pour prédire la productivité en calices. La valeur monétaire des calices par pied de $B$. costatum a été estimée à partir de la valeur d'échange des calices secs sur les marchés en trois périodes de l'année. Les résultats montrent que la productivité en calices n'est pas significativement influencée par le mode d'utilisation des terres. Parmi les modèles allométriques ajustés, le modèle linéaire de la forme $\sqrt{ } Y=a+b X$ prédit le mieux la productivité en calices de $B$. costatum. La valeur monétaire des calices évaluée par pied varie suivant le diamètre et la période. Pour une même période, elle est de 2,57 \$US pour les diamètres compris entre 10 et $25 \mathrm{~cm}$ et 26,10 \$US pour les diamètres supérieurs ou égaux à $50 \mathrm{~cm}$. Sachant que la valeur monétaire des calices ne représente qu'une partie de la valeur monétaire totale de $B$. costatum, il importe de sensibiliser les populations locales à la plantation de l'espèce et à des techniques de récolte peu préjudiciables.

Mots-clés : Bombax costatum, conservation, espèce menacée, modèle allométrique, mode d'utilisation des terres, valeur d'échange, Burkina Faso.

\section{ABSTRACT}

\section{PRODUCTIVITY AND ECONOMIC VALUE OF THE BOMBAX COSTATUM PELLEGR. \& VUILLET CALYX IN BURKINA FASO'S SUDANIAN ZONE}

The silk cotton tree Bombax costatum is found in Sahelian-Sudanian and Guinean open woodland and savannah lands. It is heavily used for its calyxes and has come under threat in several areas of Burkina Faso. An alternative conservation approach would be to recognise the economic value of the species. This study produced an assessment in monetary value of $B$. costatum calyx productivity in order to support arguments for the conservation of the species. To do so, a sample of individual $B$. costatum trees was analysed according to three patterns of land use. All of the flowers were harvested in order to estimate the number of calyxes produced. The monetary value of the calyxes from each $B$. costatum tree was estimated from local market prices for dry calyxes during three periods of the year. The results show that calyx productivity is not significantly influenced by the pattern of land use. Among the adjusted allometric models used, only the linear $\sqrt{ } Y=a+b X$ model provided a reliable forecast of $B$. costatum calyx productivity. The estimated monetary value of the calyxes from each tree varied with their diameter and with the time of year. Over the same period, their value varied from 2.57 US\$ for diameters of $10-25 \mathrm{~cm}$ and 26.10 US\$ for diameters of $50 \mathrm{~cm}$ and more. Given that the monetary value of the calyxes represents only a part of the total monetary value of $B$. costatum, it is important to raise awareness locally on planting the species and using less destructive harvesting techniques.

Keywords: Bombax costatum, conservation, threatened species, allometric model, land use pattern, market value, Burkina Faso.

\section{RESUMEN}

\section{PRODUCTIVIDAD Y VALOR ECONÓMICO DE CÁLICES DE BOMBAX COSTATUM PELLEGR. \& VUILLET EN LA ZONA SUDANESA DE BURKINA FASO}

Árbol de las sabanas de los bosques abiertos de la zona climática sahelianosudanesa a guineana, Bombax costatum es una especie que sufre una intensa explotación por sus cálices y se halla amenazada en varias localidades de Burkina Faso. Una alternativa a la conservación consistiría en reconocer el interés económico de la especie. Este estudio evalúa el valor monetario de la productividad en cálices de $B$. costatum para aportar argumentos necesarios para su conservación. Para ello, se muestrearon individuos de $B$. costatum en tres modos de uso de la tierra y se cosechó totalmente la producción floral para evaluar la cantidad de cálices producida. Se elaboraron modelos alométricos para predecir la productividad de cálices. El valor monetario de los cálices por pie de $B$. costatum se estimó a partir del valor de cambio de los cálices secos en los mercados en tres períodos del año. Los resultados muestran que la productividad de cálices no se ve influida significativamente por el modo de uso de la tierra. Dentro de los modelos alométricos ajustados, sólo el modelo lineal de la forma $\sqrt{ } Y=a+b X$ predice adecuadamente la productividad de cálices de $B$. costatum. El valor monetario de los cálices, evaluado por pie, varía según el diámetro y el período. En un mismo período, este valor es de 2.57 \$ US en diámetros de 10 a $25 \mathrm{~cm}$ y de 26.10 \$ US en los diámetros superiores o iguales a $50 \mathrm{~cm}$. Teniendo en cuenta que el valor monetario de los cálices sólo representa una parte del valor monetario total de $B$. costatum, es importante concienciar a las poblaciones locales para que planten esta especie y utilicen técnicas de cosecha poco dañinas.

Palabras clave: Bombax costatum, conservación, especie amenazada, modelo alométrico, modo de uso de la tierra, valor de cambio, Burkina Faso. 


\section{Introduction}

Au Burkina Faso, les populations rurales dépendent étroitement des ressources végétales pour leurs besoins en alimentation, fourrage, bois énergie, pharmacopée, artisanat et autres produits (BELEM et al., 2007 ; SOP et al., 2012). Dans le domaine de l'alimentation, certaines espèces sont utilisées comme compléments alimentaires (KOUYATÉ, LAMIEN, 2009 ; MERTZ et al., 2001) et d'autres en période de soudure. Les organes de ces espèces sont vendus en zones rurales et urbaines et génèrent des revenus monétaires. C'est le cas des fruits de Tamarindus indica, Detarium microcarpum et Saba senegalensis, des amandes de Vitellaria paradoxa et Parkia biglobosa, des feuilles de Adansonia digitata, mais aussi des calices de Bombax costatum (KOUYATÉ et al., 2006).

Bombax costatum Pellegr. \& Vuillet de la famille des Malvaceae est un arbre de 10 à $25 \mathrm{~m}$ de haut des savanes et forêts claires sahélo-soudaniennes à guinéennes, se rencontrant sur de nombreux types de sols mais très souvent sur des stations latéritiques ou rocheuses. L'espèce est représentée par des individus généralement dispersés, mais parfois grégaires, pour former des peuplements dans les formations naturelles et anthropisées (champs et jachères). Connue sous l'appellation de «kapokier à fleurs rouges », elle est d'une grande importance socio-économique. Ses feuilles sont utilisées comme fourrage, son bois en artisanat, son écorce et ses feuilles en pharmacopée. Les calices des fleurs sont utilisés à l'état frais ou sec dans la préparation d'une sauce très appréciée. Les calices sont récoltés en décembre-janvier et consommés immédiatement ou séchés pour être stockés. Ils sont également commercialisés (BELEM et al., 2007, 2008). Dans plusieurs localités au Burkina Faso, le kapokier à fleurs rouges fait partie des espèces préférées des populations (MERTZ et al., 2001) et est menacé à cause d'une surexploitation de ses différents organes. La consommation des calices prive les adultes de leur potentiel semencier (BELEM et al., 2008). Son intérêt pour les populations locales et ces menaces font de Bombax costatum une espèce à conserver en priorité (SOP et al., 2012).

Au Burkina Faso, outre les travaux qui ont documenté l'impact de ces usages sur $B$. costatum, d'autres ont proposé des stratégies pour sa conservation. BELEM et al. (2008) ont mis au point des techniques pour stimuler, contrôler et optimiser la capacité de drageonnage de l'espèce. OUÉDRAOGO et THIOMBIANO (2012) ont montré que $B$. costatum se renouvelle d'environ $95 \%$ par drageonnage, avec une structure de populations juvéniles relativement satisfaisante. Pendant la saison sèche, les jeunes plants sont cependant confrontés à une forte mortalité. Ces auteurs ont suggéré la plantation, l'entretien et la protection de jeunes plants pour conserver l'espèce. Ces recommandations n'ont pas encore été suivies d'actions, sans doute parce que les retombées économiques et monétaires ne sont pas connues (COLON et al., 2009). La
Convention sur la diversité biologique recommande fortement le recours à l'argumentaire économique pour la préservation de l'environnement. Les techniques d'évaluation économique de la biodiversité sont cependant complexes et controversées (BRAHIC, TERREAUX, 2009) ; toutefois, elles s'accordent sur la nécessité d'inventorier au préalable les services écosystémiques. L'évaluation économique des services d'approvisionnement est relativement aisée dans la mesure où ces services sont largement échangés sur les marchés (BRAHIC, TERREAUX, 2009). Aussi, cette évaluation gagnerait à se focaliser d'abord sur les retombées des fonctions de production écologique pour arriver à définir une valeur monétaire. Il est urgent, dans ce contexte, de quantifier la productivité des espèces végétales constitutives des écosystèmes forestiers, en l'occurrence celles qui présentent un intérêt socio-économique avéré pour les populations. Or, les perturbations anthropiques relevant des divers modes d'utilisation des terres influencent significativement la productivité des espèces végétales (SCHUMANN et al., 2010). L'évaluation de la productivité selon le mode d'utilisation des terres constitue dès lors une étape de base pour évaluer la valeur monétaire de l'espèce.
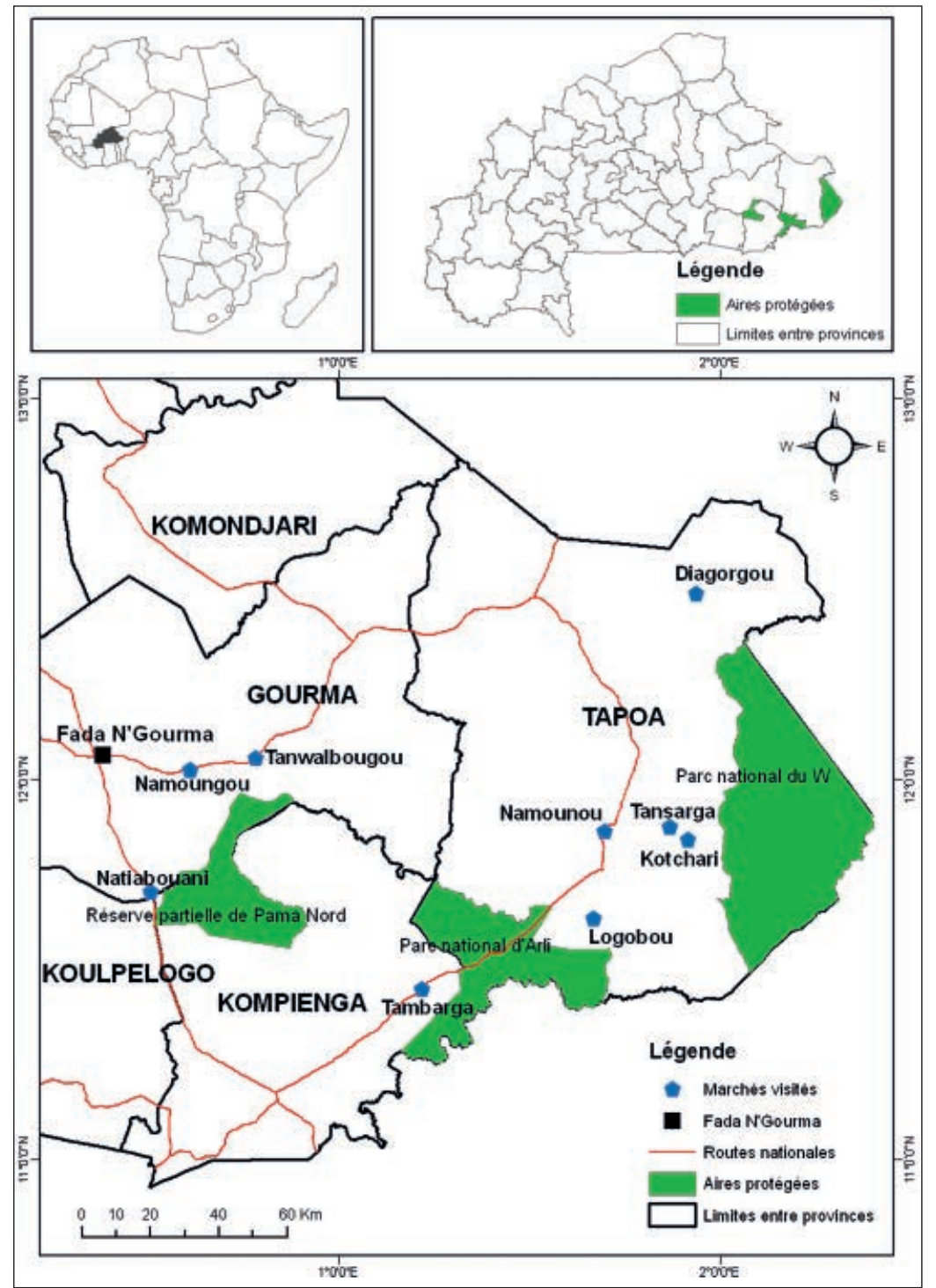

Figure 1.

Localisation de la zone d'étude. 


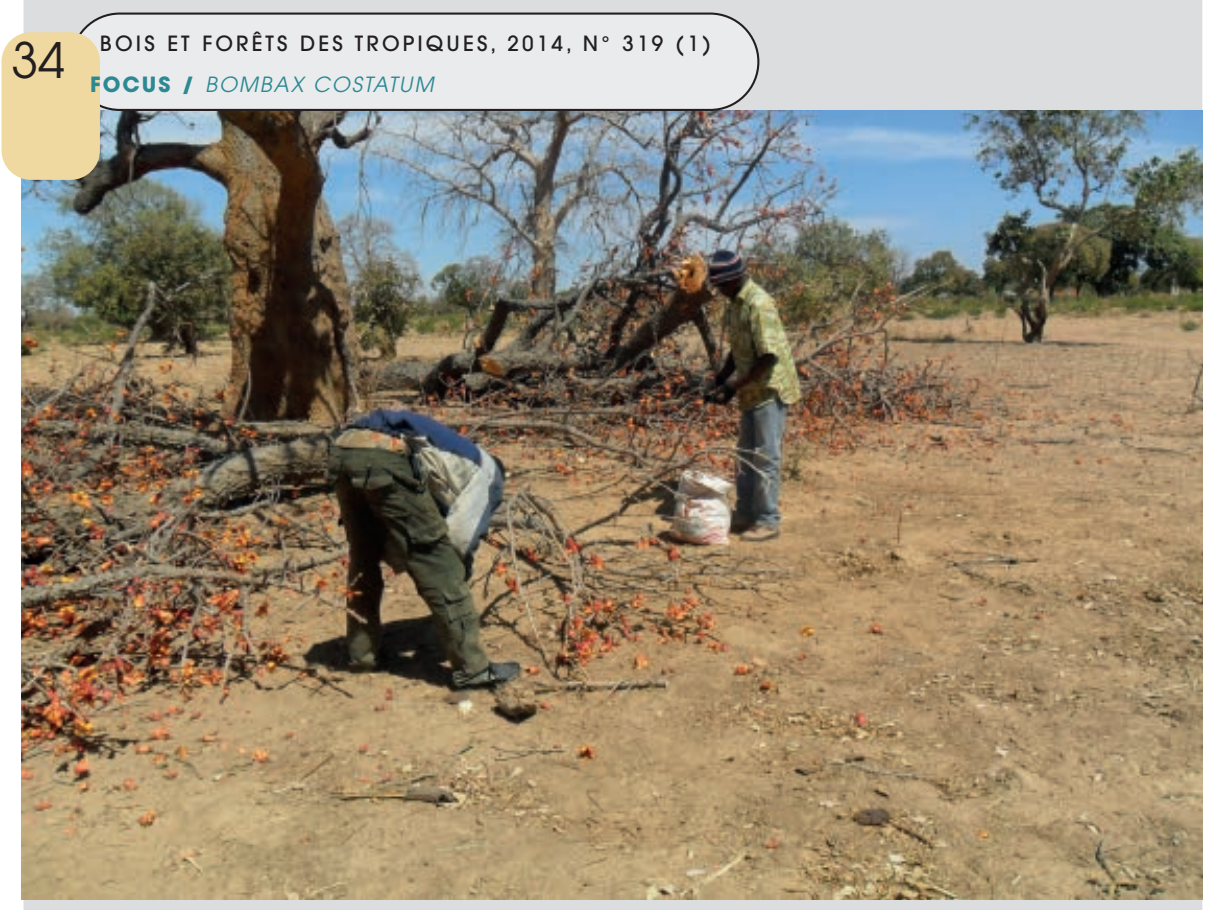

Photo 2.

Ramassage de fleurs dans un champ.

Photo I. Ouédraogo.

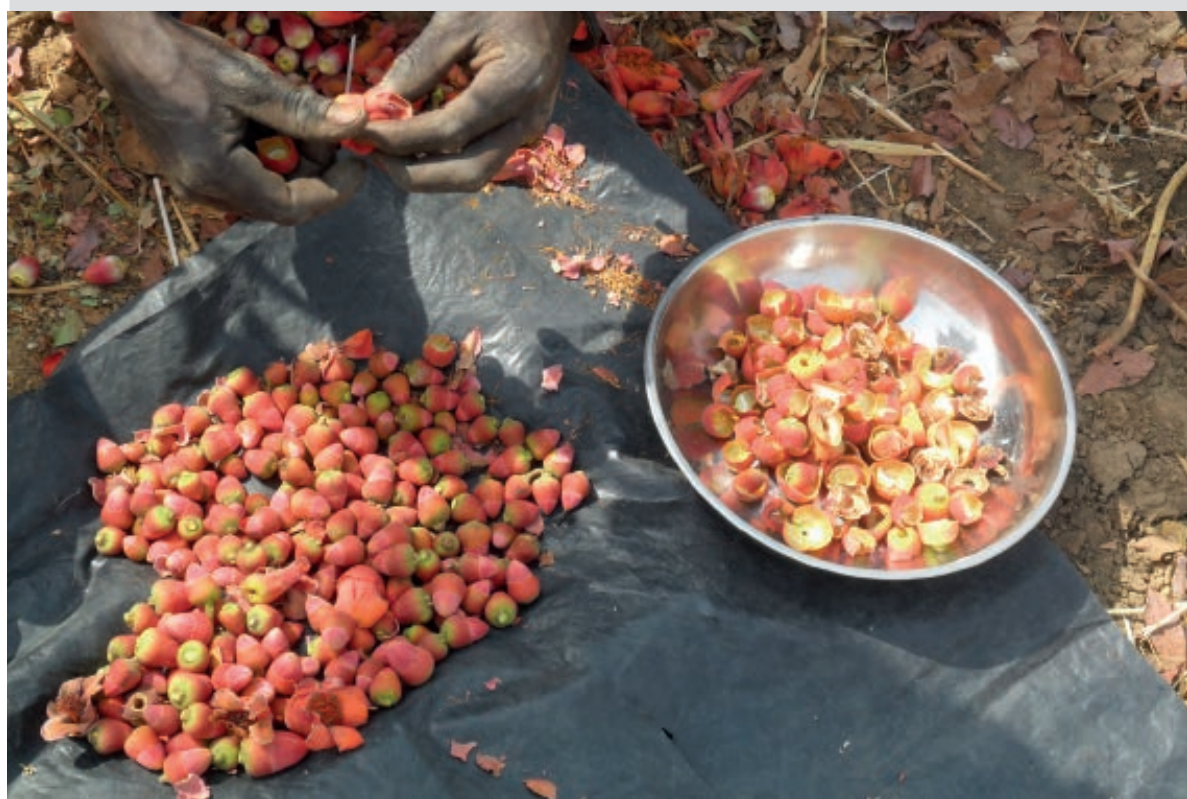

Photo 3.

Récolte des calices pour pesage.

Photo I. Ouédraogo.

\section{Matériel et méthodes}

\section{Milieu d'étude}

La zone d'étude recouvre le parc national du W (Pnw), le parc national d'Arli (Pna) et la réserve partielle de faune de Pama Nord (Rpfpn), situés dans la région Est du Burkina Faso. Les données ont été collectées d'une part dans trois modes d'utilisation des terres (aires protégées, zones villageoises d'intérêts cynégétiques, champs) et d'autre part dans les marchés riverains des aires protégées (figure 1).

Les aires protégées subissent des feux précoces d'aménagement entre novembre et décembre. En outre, selon le code forestier du Burkina Faso, les droits d'usage traditionnels sont maintenus pour les populations riveraines. Ils concernent le ramassage du bois mort, la cueillette des fruits mûrs et la récolte des produits médicinaux. Les mêmes droits d'usage s'appliquent aux zones villageoises d'intérêts cynégétiques (Zovic), aires protégées gérées par les communautés de base avec l'assistance des services techniques locaux. Ces espaces de brousse sont préservés de l'agriculture et du déboisement. La chasse traditionnelle et le pâturage domestique y sont prohibés alors que dans les champs le pâturage, les feux incontrôlés et l'exploitation des produits forestiers ligneux et non ligneux de subsistance y sont des pratiques courantes (BELEM et al., 2007).

Les zones anthropisées sont moins riches en espèces végétales que les aires protégées. Ces dernières sont des zones de refuge d'espèces à haute valeur socio-économique. Les espèces végétales ligneuses les plus fréquentes des aires protégées sont Anogeissus leiocarpa, Terminalia avicennioides, Acacia dudgeoni, Acacia hockii, Crossopteryx febrifuga, Combretum nigricans, Acacia gourmaensis et Combretum glutinosum. Elles sont caractéristiques des formations naturelles de savane et dominées par des espèces de la famille des Combretaceae, avec des individus de diamètre le plus souvent inférieur à $50 \mathrm{~cm}$. Cet ensemble est régi

Cette étude a pour objet de fournir des informations sur la valeur monétaire des calices produits par $B$. costatum en vue d'inciter les décideurs et les bailleurs de fonds à sa gestion durable. Pour ce faire, il s'est agi d'évaluer la quantité annuelle de calices produite par $B$. costatum suivant le mode d'utilisation des terres, de développer un modèle allométrique pour prédire la productivité en calices et d'évaluer les variations de la valeur monétaire des calices suivant la période et la localité. par un climat de type soudanien avec une saison pluvieuse d'avril à octobre, que suit une saison sèche de novembre à mars. La moyenne pluviométrique annuelle est comprise entre $750 \mathrm{~mm}$ et $950 \mathrm{~mm}$ (FONTES, GUINKO, 1995).

La région de l'Est du Burkina Faso est peuplée d'environ 1200000 habitants, soit une densité moyenne de 26 habitants par km² (INSD, 2008). La périphérie des aires protégées est occupée par plusieurs groupes ethniques dont les principaux sont les Gourmantché, les Mossé, les Peuls et les Djerma. Ils vivent principalement d'agriculture et d'élevage. 


\section{Collecte des données}

\section{Échantillonnage des arbres}

Pour une meilleure prédiction de la productivité, la collecte de l'information sur la biomasse des arbres et arbustes doit prendre en compte les grands et petits diamètres (MBOW et al., 2013). Trois classes de diamètre incluant les petits diamètres, les diamètres intermédiaires et les grands diamètres ont ainsi été définies. Sur la base du plus petit diamètre $(10,3 \mathrm{~cm})$ productif de $B$. costatum constaté lors de la collecte de données, 66 individus échantillonnés ont été répartis dans les trois classes de diamètre. La classe 1 regroupe 16 arbres de diamètre compris entre 10 et $25 \mathrm{~cm}$; la classe 2 recouvre 34 individus dans l'intervalle de diamètre $25-50 \mathrm{~cm}$; et la classe 3 rassemble 16 individus d'un diamètre supérieur ou égal à $50 \mathrm{~cm}$. Les arbres ont été échantillonnés de manière à minimiser l'interdépendance des observations. C'est-à-dire que les arbres sont échantillonnés de sorte que les caractéristiques d'un arbre n'influencent pas les caractéristiques d'un autre. Les principaux critères de choix des individus ont porté sur leur disponibilité et l'absence de traces d'élagage ou d'ébranchage. Ce principe de choix rend compte de la productivité potentielle de l'espèce ; sa productivité lorsque celle-ci n'a pas subi une exploitation humaine.

\section{Mesures dendrométriques et évaluation de la productivité}

Les calices de $B$. costatum ont été récoltés au stade de floraison maximale, entre les mois de décembre et janvier, dans les trois modes d'utilisation des terres. La production florale des pieds a été intégralement récoltée (photos 1 et 2) et pesée. Cette méthode par élagage ponctuel offre l'avantage d'avoir des données plus proches de la réalité et permet à l'arbre de rejeter l'année suivante. Pour éviter une confusion des fleurs du pied échantillonné avec celles d'autres pieds voisins, les pieds isolés ont été préférés au détriment des peuplements. Sur chaque individu, un échantillon de $1 \mathrm{~kg}$ de fleurs fraîches a été récolté et les calices qui représentent les parties comestibles ont été séparés du reste de la fleur (corolle, étamines et pistil) (photo 3). Les calices obtenus ont été pesés directement sur le terrain puis ramenés au laboratoire pour déterminer le poids sec après séchage à l'étuve.

Les paramètres dendrométriques mesurés avant la récolte sont le diamètre $\mathrm{D} 20(\mathrm{~cm})$ à $0,20 \mathrm{~m}$ du sol, le diamètre $\operatorname{Dhp}(\mathrm{cm})$ à 1,30 $\mathrm{m}$ du sol, le petit diamètre Dh1 (m) et le grand diamètre Dh2 $(\mathrm{m})$ du houppier, la hauteur du fût $\mathrm{Hf}$ (m) et la hauteur totale $\mathrm{Ht}(\mathrm{m})$ de l'arbre (figure 2). Pour les individus multicaules, les D20 et Dhp ont été mesurés sur chaque tige.

\section{Enquêtes de marché}

Des enquêtes de marché ont été conduites pour évaluer la valeur d'échange que représente le prix des calices secs. En effet, les calices sont conservés sous la forme sèche toute l'année. Les enquêtes ont été réalisées dans neuf marchés d'importance similaire (figure 1) en période froide (janvier-février), au début (juin) et à la fin (octobre) de la saison pluvieuse afin de prendre en compte la disponibi- lité du produit et la variation de sa valeur d'échange. Le poids et le prix de vente ont été relevés chez deux vendeurs pris aléatoirement dans chaque marché à chacune des périodes (photo 4). L'abondance du produit dans le marché a été appréciée sur une échelle à trois niveaux. Les calices secs sont dits " abondants sur le marché » s'il y a au moins dix vendeurs, "moyens sur le marché » si le nombre de vendeurs est compris entre cinq et neuf et «faibles sur le marché » si le nombre de vendeurs est inférieur à cinq.

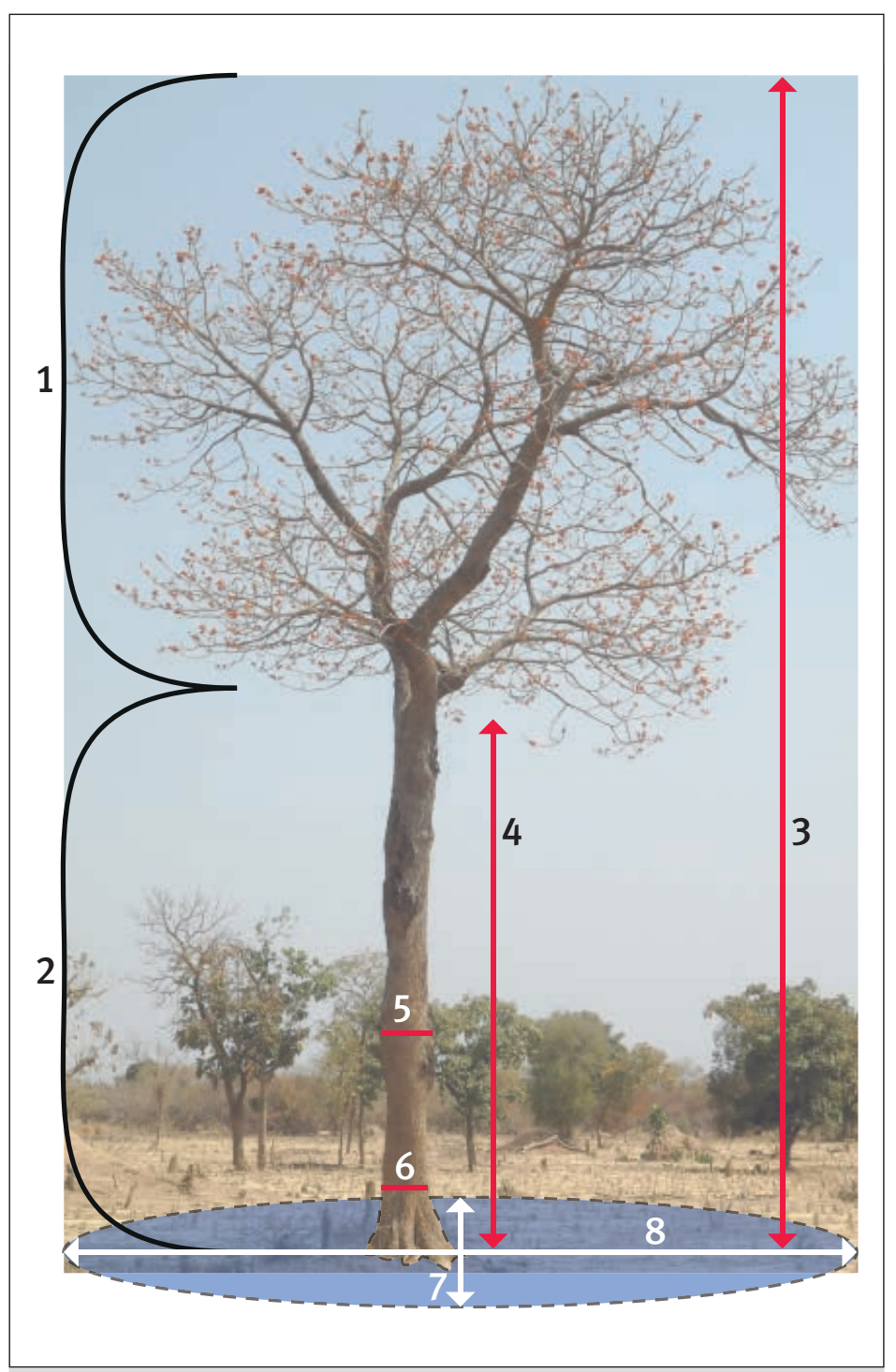

Figure 2.

Illustration de mesure des paramètres dendrométriques. 1 = houppier de l'arbre ; 2 = fût de l'arbre ; 3 = mesure de la hauteur totale $(\mathrm{Ht}(\mathrm{m})) ; 4=$ mesure de la hauteur du fût ( $\mathrm{Hf}$ $(\mathrm{m})) ; 5=$ mesure du diamètre à $1,30 \mathrm{~m}(\operatorname{Dhp}(\mathrm{cm})) ; 6=$ mesure du diamètre à $0,20 \mathrm{~m}(\mathrm{D} 20(\mathrm{~cm})) ; 7=$ mesure $\mathrm{du}$ petit diamètre du houppier $(\mathrm{Dh} 1(\mathrm{~m})) ; 8=$ mesure du grand diamètre du houppier (Dh2 (m)). 


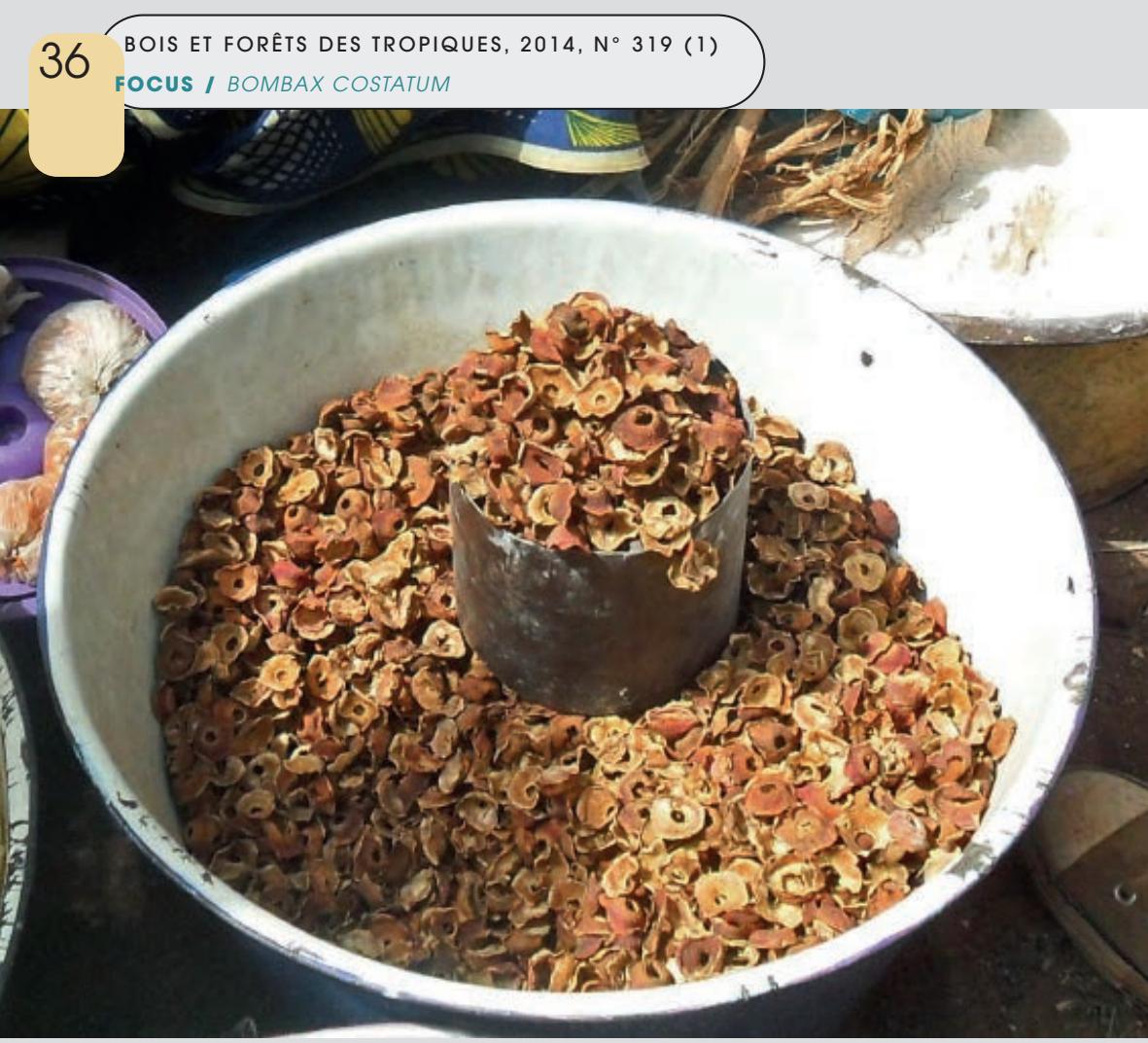

Photo 4.

Mesure des calices secs pour la vente sur un marché. Photo I. Ouédraogo.

Le test W de Shapiro-Wilk a permis de vérifier la normalité de la distribution des données de poids sec des calices (PSc). Le test non paramétrique de comparaison de moyenne de Wilcoxon/Kruskal-Wallis au seuil de $5 \%$ a été effectué entre les productions des arbres pour les différents modes d'utilisation des terres. Le poids sec total moyen des calices par pied et par an et les écarts-types ont été calculés en fonction des trois classes de diamètre.

\section{Élaboration des modèles allométriques}

Une analyse discriminante linéaire (Adl) pas à pas a été effectuée pour identifier progressivement les variables explicatives qui discriminent au mieux la productivité en calices de $B$. costatum. À cet effet, les productivités en calices des individus échantillonnés ont été réparties en six classes de poids : $0-1 \mathrm{~kg} ; 1-2 \mathrm{~kg} ; 2-3 \mathrm{~kg} ; 3$ $4 \mathrm{~kg}$; $4-5 \mathrm{~kg}$; supérieur à $5 \mathrm{~kg}$.

Parmi les 66 observations effectuées, 56 ont servi à l'ajustement des modèles. Plusieurs modèles ont été ajustés par la méthode des moindres carrés standards avec des transformations des variables explicatives et/ou de la variable expliquée, et les plus performants ont été retenus sur la base des coefficients de détermination $\mathrm{R}^{2}$ et de l'erreur résiduelle. L'ajustement d'un modèle est d'autant meilleur que ces résidus sont faibles.

\section{Analyse des données}

Le test de Mantel avec 999 permutations proposé par le logiciel PASSaGE 2 (ROSENBERG, ANDERSON, 2011) a été appliqué au préalable afin d'évaluer l'autocorrélation des individus de $B$. costatum échantillonnés. Les différents paramètres dendrométriques mesurés, la productivité des arbres échantillonnés ainsi que les coordonnés géographiques ont été utilisés à cet effet. Les coefficients $(r=-0,018 ; t=-0,33$; righttailed $p=0,63$; two-tailed $p=0,74)$ issus du test de Mantel n'étant pas significatifs, il n'y a donc pas d'autocorrélation apparente entre les individus échantillonnés.

\section{Comparaison des productivités}

Les moyennes des diamètres et le poids sec des calices ont été calculés pour l'analyse des données de productivité. Le diamètre moyen Dh du houppier a été calculé par la moyenne arithmétique des Dh1 et Dh2. Les Dhp et D20 des individus multicaules ont été déduits par la moyenne quadratique des tiges. La détermination du poids sec des calices (PSc) exprimé en kilogrammes (kg) a été obtenue sur la base de la relation:

$\mathrm{PSc}=\mathrm{PFT} \times \mathrm{psc}$

Avec :

PSc, poids sec total des calices;

PFT, poids frais total des fleurs ;

Psc, teneur en matière sèche de calices de $1 \mathrm{~kg}$ de fleurs fraîches.
L'ajustement est achevé lorsqu'il est vérifié que les résidus sont indépendants, que leur distribution est normale d'espérance nulle et qu'ils ont une variance constante (PICARD et al., 2012).

Lorsque plusieurs modèles avec le même nombre de paramètres sont ajustés, le meilleur modèle est celui avec la plus forte vraisemblance ; il présente la plus petite valeur du critère d'information d'Akaike ou AIC (PICARD et al., 2012).

Les meilleurs modèles ajustés ont été validés à partir d'une régression linéaire entre les valeurs prédites par ces modèles et les observations réelles (LUFAFA et al., 2008). À cet effet, les 10 observations restantes des 66 observations, choisies de façon aléatoire à travers les modes d'utilisation des terres, ont été utilisées. L'ensemble des données ont été analysées à l'aide du logiciel JMP 9 (SAS INSTITUTE, 2010).

\section{Évaluation des prix}

Les données d'enquête de marché ont été analysées suivant une statistique descriptive avec des calculs de paramètres de position (prix moyens). Le poids et le prix de vente des calices secs ont permis de déduire la valeur d'échange moyenne du kilogramme de calices secs de B. costatum par période dans les différents marchés en FCFA $/ \mathrm{kg}$ et $\$$ US $/ \mathrm{kg}$. La valeur d'échange moyenne du kilogramme de calices secs par période a été multipliée par la production moyenne en calices pour chaque classe de diamètre afin de déterminer la valeur monétaire par pied et par an, exprimée en FCFA et \$US. 


\section{Résultats}

\section{Productivité annuelle en calices et mode d'utilisation des terres}

Le mode d'utilisation des terres n'influence pas significativement la productivité en calices de Bombax costatum $(P=0,911)$. Ceci a conduit à l'agrégation des 66 individus échantillonnés qui ont servi à l'élaboration des équations allométriques. La productivité en calices par pied croît dans le même sens que le Dhp des individus. Pour un Dhp compris dans l'intervalle $10-25 \mathrm{~cm}$, la productivité est de $0,29 \pm 0,25 \mathrm{~kg}$. Elle est de 0,93 $\pm 0,67 \mathrm{~kg}$ pour les Dhp compris entre 25 et $50 \mathrm{~cm}$. Elle atteint 2,94 $\pm 3,01 \mathrm{~kg}$ pour un diamètre supérieur à $50 \mathrm{~cm}$ (figure 3).

\section{Modèles allométriques}

La valeur du lambda de Wilk de l'analyse discriminante linéaire est de 0,17 avec un $p$-value $<0,0001$ : les variables explicatives ont un pouvoir discriminant significatif sur la productivité en calices. L'axe 1 qui représente le Dhp avec une valeur propre de 2,89 explique à lui seul 85,8\% du pouvoir discriminant, tandis que l'axe 2 représentant le Dh avec une valeur propre de 0,30 explique seulement $9 \%$ du pouvoir discriminant.

Les différentes équations ajustées et leurs paramètres de sortie sont donnés dans le tableau I. Les équations présentées ont un coefficient de détermination $\mathrm{R}^{2}$ au moins égal à 0,60. Les meilleurs modèles à erreur résiduelle et $\mathrm{AIC}$ faibles sont :

$\sqrt{P S C}(\mathrm{~kg})=-0,051+0,025 \times \operatorname{Dhp}(\mathrm{cm})$

et $\sqrt{ } \mathrm{PSC}(\mathrm{kg})=-0,064+0,132 \times \mathrm{Dh}(\mathrm{m})$.

La probabilité $P$ égale à 0,0001 pour tous les modèles atteste que les ajustements sont significatifs.

La validation des modèles par la régression linéaire entre les poids secs de calices prédits par le modèle et les poids secs de calices observés indique une très faible

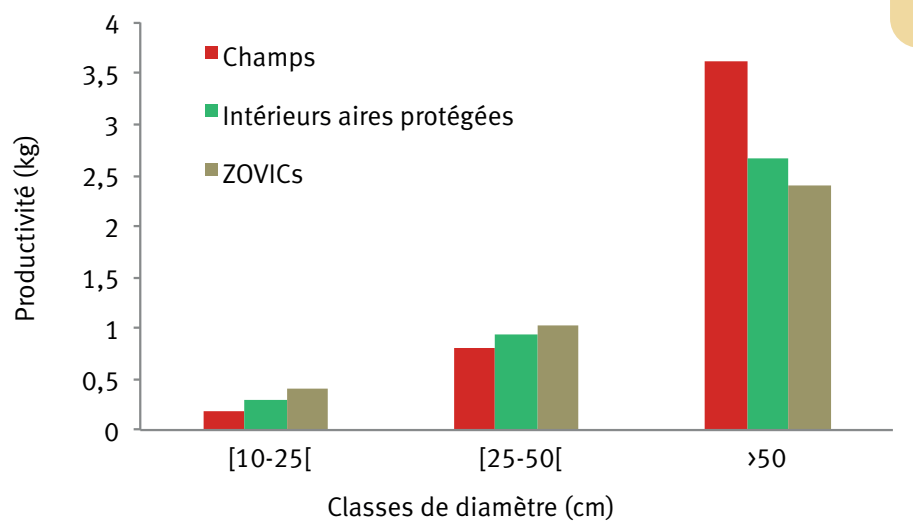

Figure 3.

Productivités en calices par classe de diamètre et par mode d'utilisation des terres.

valeur du coefficient de détermination $R^{2}(0,30)$ et une erreur résiduelle élevée $(11,25)$ pour le modèle ayant le $D h$ (m) comme variable explicative. En revanche, la valeur du coefficient de détermination $\mathrm{R}^{2}$ est forte $(0,97)$ et l'erreur résiduelle est faible $(0,43)$ pour le modèle ayant le Dhp $(\mathrm{cm})$ comme variable explicative (tableau II).

\section{Disponibilité des calices}

La disponibilité en calices secs varie suivant les localités et la période. Les calices secs ne sont pas disponibles sur tous les marchés visités en fin de saison pluvieuse (mois d'octobre). En période froide (mois de janvier à février), les calices secs sont " abondants sur le marché " de Namoungou, "moyens sur le marché » d'autres villages et « absents sur le marché » dans deux marchés à proximité du parc national du W. En début d'hivernage (mois de juin), c'est sur les marchés de Namoungou et de Tanwalbougou, aux environs de la réserve partielle de faune de Pama Nord, que les calices secs sont faiblement disponibles (tableau III).

Tableau I.

Ajustements des modèles allométriques.

\begin{tabular}{|c|c|c|c|c|c|c|}
\hline \multirow[t]{2}{*}{$\begin{array}{l}\text { Types de } \\
\text { régression }\end{array}$} & \multirow[t]{2}{*}{ Équation de la prévision } & \multicolumn{5}{|c|}{$\begin{array}{l}\text { Paramètres de sortie des modèles ajustés } \\
\text { (nombre d'observations = } 56 \text { individus) }\end{array}$} \\
\hline & & $\mathbf{R}^{2}$ & $\begin{array}{l}\text { Erreur } \\
\text { résiduelle }\end{array}$ & AIC & $\begin{array}{l}\text { Distribution } \\
\text { de la variance }\end{array}$ & $\begin{array}{l}\text { Homoscédasticité } \\
\text { de la variance }\end{array}$ \\
\hline $\begin{array}{l}\sqrt{Y}=a+b X \\
\text { avec } X=D h p\end{array}$ & $\sqrt{ } \mathrm{PSC}(\mathrm{kg})=-0,051+0,025 \times \operatorname{Dhp}(\mathrm{cm})$ & 0,71 & 5,07 & 30,85 & Acceptable & Constante \\
\hline $\begin{array}{l}\sqrt{ } Y=a+b X \\
a v e c X=D h\end{array}$ & $\sqrt{P S C}(\mathrm{~kg})=-0,063+0,132 \times \mathrm{Dh}(\mathrm{m})$ & 0,69 & 5,40 & 34,44 & Acceptable & Constante \\
\hline $\begin{array}{l}\operatorname{Ln}(Y)=a+b \operatorname{Ln}(X) \\
\operatorname{avec} X=D h\end{array}$ & $\operatorname{Ln}(\operatorname{PSc}(\mathrm{kg}))=-3,963+1,853 \times \operatorname{Ln}(\operatorname{Dh}(\mathrm{m}))$ & 0,68 & 20,30 & 108,55 & Acceptable & Constante \\
\hline $\begin{array}{l}Y=a+b X+c X^{2} \\
\text { avec } X=D h p\end{array}$ & $\operatorname{PSc}(\mathrm{kg})=-0,603+0,034 \times \operatorname{Dhp}(\mathrm{cm})+0,001 \times(\operatorname{Dhp}(\mathrm{cm}))^{2}$ & 0,84 & 29,72 & 132,23 & Acceptable & Constante \\
\hline $\begin{array}{l}Y=a+b X^{2} \\
a v e c X=D h p\end{array}$ & $\operatorname{PSc}(\mathrm{kg})=-0,231+0,0008 \times \mathrm{Dhp}^{2}\left(\mathrm{~cm}^{2}\right)$ & 0,83 & 32,44 & 134,80 & Acceptable & Constante \\
\hline
\end{tabular}


Tableau II.

Validation des meilleurs modèles allométriques ajustés.

\begin{tabular}{l|l|l}
$\begin{array}{l}\text { Types de } \\
\text { régression }\end{array}$ & Équation de la prévision & $\begin{array}{c}\text { Régressions entre valeurs prédites et observées } \\
\text { (nombre d'observations = 10 individus) } \\
\text { Erreur } \\
\text { résiduelle }\end{array}$ \\
$\mathbf{P}$
\end{tabular}

\section{Tableau III.}

Disponibilité des calices dans les marchés.

\begin{tabular}{|c|c|c|c|}
\hline Localités & $\begin{array}{c}\text { Début hivernage } \\
\text { (juin) }\end{array}$ & $\begin{array}{l}\text { Période froide } \\
\text { (janvier-février) }\end{array}$ & $\begin{array}{l}\text { Fin hivernage } \\
\text { (octobre) }\end{array}$ \\
\hline Diagorgou (W) & - & - & - \\
\hline Kotchari (W) & - & M & - \\
\hline Logobou (Arli) & - & M & - \\
\hline Namoungou (Pama Nord) & $\mathrm{F}$ & A & - \\
\hline Namounou (Arli) & - & M & - \\
\hline Natiabouani (Pama Nord) & - & M & - \\
\hline Tambarga (Arli) & - & $M$ & - \\
\hline Tansarga (W) & - & - & - \\
\hline Tanwalbougou (Pama Nord) & $\mathrm{F}$ & M & - \\
\hline
\end{tabular}

\section{Valeurs d'échange moyennes des calices}

Le kilogramme de calices secs est échangé en période froide (janvier à février) à 375 FCFA, soit 0,74 \$US, à proximité du parc national du W ; à 472 FCFA, soit 0,93 \$US, à proximité du parc national d'Arli ; à 1494 FCFA, soit 2,96 \$US, à proximité de la Rpfpn. En début d'hivernage, les calices secs sont uniquement échangés à proximité de la Rpfpn à 4484 FCFA, soit 8,88 \$US, le kilogramme.

En considérant l'ensemble de la zone d'étude, la valeur d'échange moyenne du kilogramme de calices secs de $B$. costatum varie en fonction de la période. La valeur d'échange moyenne du kilogramme de calices secs en début d'hivernage (mois de juin) est de 3,8 fois supérieure à celle en période froide (janvier à février). Elle est de 1166 FCFA, soit 2,31 \$US, en période froide contre 4484 FCFA, soit 8,88 \$US, en début d'hivernage.

\section{Valeur monétaire des calices par classe de diamètre}

La productivité annuelle en calices d'un pied de $B$. costatum échangé sur le marché produit une valeur monétaire variant selon le diamètre de l'individu et la période de l'année. Un individu à Dhp compris dans l'intervalle $10-25 \mathrm{~cm}$ a une valeur monétaire de 338 FCFA, soit 0,67 \$US, en période froide (janvier à février) ; 1300 FCFA, soit 2,57 \$US, en début d'hivernage (mois de juin). Lorsque son Dhp est compris dans l'intervalle $25-50 \mathrm{~cm}$, sa valeur monétaire atteint 1085 FCFA, soit 2,15 \$US, en période froide et 4170 FCFA, soit 8,26 \$US, en début d'hivernage. La valeur monétaire passe à 3429 FCFA, soit 6,79 \$US, en période froide et à 13184 FCFA, soit 26,10 \$US, en début d'hivernage pour un individu d'au moins $50 \mathrm{~cm}$ de diamètre (tableau IV). 


\section{Tableau IV.}

Valeur monétaire des calices par classe de diamètre et par période.

\begin{tabular}{|l|c|c|}
$\begin{array}{l}\text { Classes de } \\
\text { diamètre } \mathbf{( c m )}\end{array}$ & $\begin{array}{c}\text { Période } \\
\text { froide }\end{array}$ & $\begin{array}{c}\text { Début } \\
\text { hivernage }\end{array}$ \\
\hline $10-25[$ & $338 \mathrm{FCFA} /$ & $1300 \mathrm{FCFA} /$ \\
& 0,67 \$US & 2,57 \$US \\
\hline$[25-50[$ & $1085 \mathrm{FCFA} /$ & $4170 \mathrm{FCFA} /$ \\
& 2,15 \$US & 8,26 \$US \\
\hline$>50$ & $3429 \mathrm{FCFA} /$ & $13184 \mathrm{FCFA} /$ \\
& 6,79 \$US & 26,10 \$US \\
\hline
\end{tabular}

\section{Discussion}

\section{Productivité en calices}

Le mode d'utilisation des terres n'influence pas significativement la productivité en calices de $B$. costatum. Cependant, la productivité de certaines espèces est influencée par le mode d'utilisation des terres (SCHUMANN et al., 2010). Ces auteurs ont observé que l'émondage combiné au diamètre à $1,30 \mathrm{~m}$ de hauteur influence significativement la production fruitière de Adansonia digitata. Il est pourtant reconnu que les calices de $B$. costatum sont intensément récoltés par élagage (BELEM et al., 2007). Les résultats de cette étude s'expliquent alors par le fait que les calices de $B$. costatum sont peu consommés par la population riveraine des Pnw et Pna contrairement à celle de la Rpfpn. Cette faible exploitation des calices préserve les individus de $B$. costatum des traces d'exploitation humaine à l'intérieur des champs et des Zovic des Pnw et Pna. À l'intérieur de ces modes d'utilisation des terres, les individus de $B$. costatum restent donc sans traces d'exploitation tout comme dans les aires protégées. Également, la méthode d'échantillonnage n'a pas pris en compte les individus présentant des traces d'exploitation humaine.

Dans cette étude, seul le Dhp des arbres influe sur la productivité en calices de $B$. costatum. Ces résultats corroborent ceux de KOUYATÉ et al. (2006), qui ont montré une corrélation positive entre la productivité en fruits de Detarium microcarpum et son diamètre à $1,30 \mathrm{~m}$ de hauteur. MBOW et al. (2013) mentionnent également que les individus à petit diamètre contribuent moins à la biomasse que les individus à grand diamètre.

\section{Modèles allométriques}

La productivité en calices de $B$. costatum a été prédite par l'ajustement des modèles logarithmique, polynomial d'ordre 2 et linéaire. L'adjectif linéaire signifie que le modèle dépend linéairement de ses coefficients. Les modèles $\sqrt{ } Y=a+b X$ et $Y=a+b X^{2}$ sont des modèles linéaires car la variable réponse $Y$ dépend linéairement des coefficients a et b (PICARD et al., 2012). Le meilleur modèle est celui qui a transformé la variable réponse en racine carrée. Il est suivi par le modèle logarithmique et le modèle polynomial. Dans la littérature, deux types de modèles ressortent fréquemment pour prédire la biomasse des arbres: le modèle puissance et le modèle polynomial. Le modèle puissance est généralement linéarisé par une «log-transformation » (PICARD et al., 2012).

Le premier objectif dans l'élaboration d'une équation allométrique est son applicabilité pour l'évaluation de la biomasse des espèces de manière non destructive. L'usage du modèle utilisant le paramètre Dhp $(\mathrm{cm})$ comme variable explicative fournirait alors des valeurs en calices plus proches de la réalité car, dans plusieurs localités, $B$. costatum est exploitée pour ses calices par élagage. Il existe une possibilité de biaiser les estimations si le Dh (m) est utilisé comme variable explicative. Lorsqu'un arbre est exploité par émondage ou par ébranchage, la surface de son houppier est directement réduite alors que la circonférence n'est pas affectée (BOGNOUNOU et al., 2008).

Le $\mathrm{Dh}(\mathrm{m})$ serait en outre un paramètre inapproprié car la surface du houppier d'un arbre a tendance à s'élargir lorsqu'il est isolé et que la compétition pour la lumière est faible. Cela est fréquent au sein des espèces de parcs agroforestiers, comme c'est le cas de $B$. costatum. Ce fait expliquerait pourquoi la validation par la régression linéaire entre les poids secs de calices prédits et observés a donné une faible valeur du coefficient de détermination dans le modèle où le Dh (m) a été utilisé comme variable explicative.

Parmi les modèles de prédiction élaborés, le modèle $\sqrt{ }$ PSC $(\mathrm{kg})=-0,051+0,025 \times$ Dhp $(\mathrm{cm})$ est le plus fiable. II peut être utilisé dans des conditions pluviométriques similaires à la zone d'étude (750 mm à $950 \mathrm{~mm}$ par an) pour évaluer de manière non destructive la productivité en calices de $B$. costatum.

\section{Fixation des prix et valeur monétaire des calices}

La période froide correspond au pic de présence des calices secs dans les marchés. Cela est lié à la phénologie de l'espèce. La floraison a lieu entre décembre et février, c'est-à-dire pendant la période froide. Cependant, l'abondance des calices secs dans les marchés de Namoungou et sa valeur d'échange au kilogramme élevée démontreraient sa forte consommation par les riverains de la Rpfpn. Au cours des travaux de terrain à l'intérieur des champs et zones villageoises d'intérêts cynégétiques (Zovic) de cette aire protégée, une forte collecte des calices a été constatée. Plusieurs individus rencontrés présentaient des traces de récoltes antérieures.

L'absence des calices secs dans les marchés en fin de saison hivernale (mois d'octobre) s'explique par l'épuisement des anciens stocks. De décembre à octobre, l'ancien stock a eu le temps de s'épuiser face aux multiples sollicitations. Selon MERTZ et al. (2001), les calices de B. costatum sont présents en période sèche et la conservation jusqu'en période hivernale est rarement pratiquée. La diminution du 
stock entraîne l'augmentation de la valeur d'échange en début d'hivernage (mois de juin). Pour ces auteurs, cette diminution du stock, couplée à une demande urbaine toujours forte, entraînerait un accroissement de la valeur d'échange pendant la saison pluvieuse. Nos résultats corroborent ceux de NDOYE et al. (1997), qui ont mentionné que les prix des produits forestiers non ligneux dépendent pour beaucoup du rapport entre l'offre et la demande. Cette loi s'applique également au marché des feuilles sèches de baobab, Adansonia digitata (LAMIEN, TRAORÉ, 2003).

La valeur d'échange au kilogramme des calices secs de $B$. costatum est importante. Elle varie entre 2,31 \$US et 8,88 \$US en moyenne sur les marchés locaux de la zone d'étude. Cette valeur d'échange est supérieure à celle des feuilles sèches de $A$. digitata échangées entre 0,097 et 0,114 \$US/kg (LAMIEN, TRAORÉ, 2003) et des fruits de Detarium microcarpum dans les marchés locaux $(0,01$ à $0,05 \$ \cup S / \mathrm{kg}$ ) et régionaux $(1,61 \$ \mathrm{US} / \mathrm{kg}$ ) (KOUYATÉ, LAMIEN, 2009).

Malheureusement, la valeur monétaire que génèrent les calices de $B$. costatum reste méconnue des gestionnaires et des décideurs politiques. Elle est négligée et n'est pas prise en compte de façon spécifique dans les plans de développement et de lutte contre la pauvreté. À l'état naturel, $B$. costatum pourrait pourtant être assimilée à un facteur de production. Toute dégradation de cette espèce induit une perte de production du capital naturel qu'elle représente. L'investissement dans ce capital naturel pourrait à la fois présenter un intérêt économique et avoir un sens politique.

\section{Conclusion et implications pour la conservation}

La productivité annuelle en calices de Bombax costatum ne varie pas significativement selon le mode d'utilisation des terres. Cependant, elle dépend du niveau de croissance des arbres. Cette étude a développé un modèle allométrique pour prédire la productivité en calices de $B$. costatum et assigné une valeur monétaire aux calices. Plusieurs modèles allométriques ont été ajustés dont un seul, basé sur une transformation de la variable expliquée en racine carrée, a été retenu. La forte présence des calices secs dans les marchés et la valeur d'échange au kilogramme élevée témoignent d'une forte demande. Le marché des calices secs est régulé par la loi économique de l'offre et de la demande. Leur valeur échangée sur les marchés locaux est élevée. Cette valeur est probablement plus élevée dans les agglomérations urbaines où la demande est encore plus forte. La valeur monétaire des calices que peut générer un pied de B. costatum est cependant méconnue. Toutefois, la valeur de l'espèce ne se résume pas à la seule valeur monétaire de ses calices car $B$. costatum est une espèce à usages multiples qui procure de nombreux services écosystémiques. Donner une valeur monétaire à la biodiversité permet de comparer directement ses bénéfices aux valeurs économiques des options alternatives d'usage des ressources (BRAHIC, TERREAUX, 2009).
Cette étude a proposé des arguments pour justifier la nécessité de conserver $B$. costatum. Au regard de sa valeur évaluée et non évaluée, il est impérieux de préserver l'espèce pour les générations futures. Il faudrait alors sensibiliser les populations locales à la plantation de l'espèce et à des méthodes de récolte moins destructives. Il faudra donc abandonner la récolte continue par élagage au profit d'une récolte à l'aide de perches qui présente l'avantage d'épargner certaines fleurs vouées à devenir des semences. Cette méthode de récolte permettra d'assurer à long terme le maintien de la diversité génétique qui relève de la seule reproduction sexuée. Des travaux devront être conduits afin d'établir un seuil de récolte en deçà duquel la productivité de l'espèce n'est pas significativement influencée par l'exploitation.

\section{Remerciements}

Les auteurs remercient le Programme Loewe Centre Research Biodiversity and Climate pour avoir financé les travaux. Ils remercient également les gestionnaires des trois aires protégées et toutes leurs équipes ainsi que la population riveraine des aires protégées de la région de l'Est du Burkina Faso pour leur collaboration.

\section{Références bibliographiques}

BELEM B., BOUSSIM I. J., BELLEFONTAINE R., GUINKO S., 2008. Stimulation du drageonnage de Bombax costatum par blessure des racines au Burkina Faso. Bois et Forêts des Tropiques, $295:$ 71-79.

BELEM B., NACOULMA B. M. I., GBANGOU R., KAMBOU S., HANSEN H. H., GAUSSET Q., LUND S., RAEBILD A., LOMPO D., OUÉDRAOGO M., THEILADE I., BOUSSIM J. I., 2007. Use of Non Wood Forest Products by local people bordering the "Parc National Kaboré Tambi", Burkina Faso. The Journal of Transdisciplinary Environmental Studies, 6 (1): 1-21.

BOGNOUNOU F., SAVADOGO M., BOUSSIM I. J., GUINKO S., 2008. Équations d'estimation de la biomasse foliaire de cinq espèces ligneuses soudaniennes du Burkina Faso. Sécheresse, 19 : 201-205.

BRAHIC E., TERREAUX J.-P., 2009. Évaluation économique de la biodiversité. Méthodes et exemples pour les forêts tempérées. Paris, France, Éditions Quæ, 199 p.

COLON M., MATTERSDORF G., PAVAGEAU C., 2009. La place de l'évaluation économique de la biodiversité et des services écosystémiques dans les processus de décision. Nancy, France, AgroParisTech/IDDRI, 62 p. 
FONTES J., GUINKO S., 1995. Carte de la végétation et de l'occupation du sol du Burkina Faso. Notice explicative. Ministère de la Coopération française, Projet Campus (88 313 101), Toulouse, France, 67 p.

INSD, 2008. Recensement général de la population et de l'habitation (RGPH) de 2006 du Burkina Faso. Résultats définitifs. Ouagadougou, Burkina Faso, Ministère de l'Économie et des Finances, 52 p.

KOUYATÉ A. M., LAMIEN N., 2009. Detarium microcarpum, Sweet detar, In: SAFORGEN Priority Food Tree Species Leaflets, $8 \mathrm{p}$.

KOUYATE A. M., VAN DAMME P., DIAWARA M., 2006. Évaluation de la production en fruits de Detarium microcarpum Guil. \& Perr au Mali. Fruits, 61: 267-272.

LAMIEN N., TRAORÉ S., 2003. Commercialisation des produits non ligneux des arbres de la zone semi-aride du Burkina Faso : cas des feuilles sèches de baobab (Adansonia digitata L.). In : Bonneville J., Olivier A., Traoré C. O. $2^{\mathrm{e}}$ Atelier régional sur les aspects socio-économiques de l'agroforesterie au Sahel, Bamako, Mali, 4-6 mars 2002. Comptes rendus Icraf, Bamako, Mali, Université Laval, Québec, Canada, 11-19.

LUFAFA A., DIÉDHIOU I., SAMBA S. A. N., SÉNÉ M., KHOUMA M., KIZITO F., DICK R. P., DOSSA E., NOLLER J. S., 2008. Carbon stocks and patterns in native shrub communities of Senegal's Peanut Basin. Geoderma, 146: 75-82.

MBOW C., VERSTRAETE M. M., SAMBOU B., DIAW T. A., NEUFELDT H., 2013. Allometric models for aboveground biomass in dry savanna trees of the Sudan and SudanGuinean ecosystems of Southern Senegal. Journal of Forest Research, Doi 10.1007/s10310-013-0414-1.

MERTZ O., LYKKE A. M., REENBERG A., 2001. Importance and seasonality of vegetable consumption and marketing in Burkina Faso. Economic Botany, 55: 276-289.

NDOYE O., RUIZ PÉREZ M., EYEBE A., 1997. Les marchés des produits forestiers non ligneux dans la zone de forêt humide du Cameroun. Londres, Royaume-Uni, Overseas Development Institute, Réseau foresterie pour le développement rural, Document 22c, 20 p.

OUÉDRAOGO A., THIOMBIANO A., 2012. Regeneration pattern of four threatened tree species in Sudanian savannas of Burkina Faso. Agroforestry Systems, 86 (1): 35-48.

PICARD N., SAINT-ANDRÉ L., HENRY M., 2012. Manuel de construction d'équations allométriques pour l'estimation du volume et la biomasse des arbres : de la mesure de terrain à la prédiction. Cirad et Fao, 222 p.
ROSEnBERG M. S., ANDERSON C. D., 2011. PASSaGE: Pattern Analysis, Spatial Statistics and Geographic Exegesis, version 2. Methods in Ecology and Evolution, 2: 229-232.

SAS INSTITUTE INC., 2010. JMP 9 Discovering JMP. Cary, NC, États-Unis, SAS Institute, 148 p.

SCHUMANN K., WITTIG R., THIOMBIANO A., BECKER U., HAHN K., 2010. Impact of land-use type and bark- and leafharvesting on population structure and fruit production of the baobab tree (Adansonia digitata L.) in a semi-arid savanna, West Africa. Forest Ecology and Management, 260 (11): 2035-2044.

SOP T. K., OLDELAND J., BOGNOUNOU F., SCHMIEDEL U., THIOMBIANO A., 2012. Ethnobotanical knowledge and valuation of woody plants species: a comparative analysis of three ethnic groups from the sub-Sahel of Burkina Faso. Environment, Development and Sustainability, 14 (5): 627-649. 